\title{
Mitochondrial dysfunction in the liver of type 2 diabetic Goto-Kakizaki rats: improvement by a combination of nutrients
}

\author{
Jiejie $\mathrm{HaO}^{1} \dagger$, Weili Shen ${ }^{2} \dagger$, Lijuan Sun $^{3}$, Jiangang Long $^{4}$, Edward Sharman ${ }^{5}$, Xianglin Shi ${ }^{3}$ \\ and Jiankang Liu ${ }^{3,4 *}$ \\ ${ }^{1}$ Key Laboratory of Marine Drugs, Ministry of Education, School of Medicine and Pharmacy, Ocean University of China, \\ Qingdao, People's Republic of China \\ ${ }^{2}$ State Key Laboratory of Medical Genomics, Shanghai Key Laboratory of Vascular Biology, Department of Hypertension, \\ Ruijin Hospital, Shanghai Jiaotong University School of Medicine, Shanghai 200025, People's Republic of China \\ ${ }^{3}$ Graduate Center for Toxicology, College of Medicine, University of Kentucky, Lexington, KY 40536, USA \\ ${ }^{4}$ The Key Laboratory of Biomedical Information Engineering of Ministry of Education, Institute of Mitochondrial Biology and \\ Medicine, Xi'an Jiaotong University School of Life Science and Technology, Xi'an 710049, People's Republic of China \\ ${ }^{5}$ Department of Neurology, University of California, Irvine, CA 92697, USA \\ (Received 7 September 2010 - Revised 1 November 2010 - Accepted 20 January 2011 - First published online 22 March 2011)
}

\begin{abstract}
Treatment with a combination of four nutrients, i.e. $R$ - $\alpha$-lipoic acid, acetyl-L-carnitine, nicotinamide and biotin, just as with pioglitazone, significantly improves glucose tolerance, insulin release, plasma NEFA, skeletal muscle mitochondrial biogenesis and oxidative stress in Goto-Kakizaki (GK) rats. However, it is not known whether treatment with these nutrients can improve mitochondrial function and reduce oxidative stress in GK rats. The effects of a combination of these four nutrients on mitochondrial function, oxidative stress and apoptosis in GK rat liver were investigated. Livers of untreated GK rats showed (1) abnormal changes in the activities of mitochondrial complexes (decreases in I, III and IV and increases in II and V), (2) increases in protein oxidation, (3) decreases in antioxidant enzymes (superoxide dismutase, glutathione $S$-transferase, NADH-quinone oxidoreductase-1), (4) a decrease in total antioxidant capacity but increases in reduced glutathione level and glyceraldehyde 3-phosphate dehydrogenase expression and (5) significant increases in apoptosis biomarkers, including expression of p21 and p53. A 3-month treatment with the four nutrients significantly improved most of these abnormalities in GK rats, and the effects of the nutrient combination were greater than those of pioglitazone for most of these indices. These results suggest that dietary supplementation with nutrients that are thought to influence mitochondrial function may be an effective strategy for improving liver dysfunction in GK diabetic rats.
\end{abstract}

Key words: $\boldsymbol{R}$ - $\boldsymbol{\alpha}$-Lipoic acid: Acetyl-L-carnitine: Nicotinamide: Biotin: Oxidative damage: Phase II enzymes: Apoptosis

Type 2 (non-insulin-dependent) diabetes mellitus is one of the most common metabolic diseases in humans, affecting about $3 \%$ of the human population. Clinically, it is rather a group of related diseases, characterised by chronic hyperglycaemic levels, due to an impairment in the balance among the three processes of $\beta$-cell secretion of insulin, peripheral resistance to insulin and hepatic glucose production ${ }^{(1,2)}$. Depending on several factors, such as obesity, age of onset, mode of inheritance and severity of glucose intolerance, clinical features of individuals suffering from type 2 diabetes are highly variable.
Despite being one of the most common non-communicable diseases in the world, the exact mechanism (or mechanisms) leading to glucose intolerance is still not clear ${ }^{(1,3)}$. Recently, mitochondrial dysfunction due to oxidative damage has become known as a major contributor to ageing, to degenerative diseases such as cancer, and to the metabolic syndrome, obesity and type 2 diabetes ${ }^{(4,5)}$. A group of antioxidants/ metabolites which can act to improve mitochondrial function and protect mitochondria from oxidative damage, when supplemented in the diet, have been defined as mitochondrial

Abbreviations: CoQ, coenzyme quinone; CPT-1, carnitine palmitoyltransferase I; LA, lipoic acid; GAPDH, glyceraldehyde 3-phosphate dehydrogenase; GK, Goto-Kakizaki; GSH, reduced glutathione.

*Corresponding author: J. Liu, email j.liu@mail.xjtu.edu.cn

† These authors contributed equally to this work. 
nutrients ${ }^{(6,7)}$. One good example of a mitochondrial nutrient is $R$ - $\alpha$-lipoic acid (LA) ${ }^{(7-9)}$. Here, the terms nutrients or mitochondrial nutrients denote the combination of LA, acetyl-L-carnitine, nicotinamide and biotin.

The Goto-Kakizaki (GK) rat is known as a model of type 2 diabetes. The pathogenesis of diabetes in GK rats involves insulin resistance, an abnormal glucose metabolism as well as an impaired ontogenetic development of pancreatic islet cells $^{(10,11)}$ and mitochondrial dysfunction in the liver ${ }^{(12)}$. This model is particularly relevant to the understanding of human type 2 diabetes because mild hyperglycaemia has been demonstrated as early as $2-4$ weeks after birth in these animals. Until 12 months of age, GK rats exhibit a moderate and stable fasting hyperglycaemia, which does not progress to a ketotic state. Therefore, at an early stage of diabetes, the GK rat does not present the severe complications associated with this condition. It is thus an appropriate model to study events occurring at the onset of diabetes, compared with obese diabetic rats which present severe hyperglycaemia and hyperlipidaemia ${ }^{(13)}$.

Treatment of GK rats with mitochondrial nutrients significantly improves glucose tolerance, insulin release, plasma NEFA levels, skeletal muscle mitochondrial biogenesis ${ }^{(14)}$ and immune function ${ }^{(15)}$. The rationale for the selection and dosage levels of these nutrients, as detailed earlier ${ }^{(14,15)}$, targets substances that influence mitochondrial processes either synergistically or by mechanisms as widely disparate as possible. Briefly, acetyl-L-carnitine and LA were selected for the ability of each to ameliorate mitochondrial dysfunction: acetyl-L-carnitine by its ability to facilitate the transport of long-chain fatty acids across the mitochondrial membrane and LA by its ability to improve insulin sensitivity by adenosine monophosphate-activated protein kinase (AMPK) activation. Both nutrients were included because the combination is more effective than either nutrient administered alone. Niacin was added for its ability to lower plasma glucose, plasma NEFA and hepatic glucose production, and for its enhancement of insulin-stimulated glucose uptake in streptozotocin-treated rats. Finally, biotin was selected based on its role as a cofactor for four mitochondrial carboxylases, activities of which are thought to be normalised by a combination of biotin and LA.

The liver is one of the major organs affecting and being affected by diabetes and hyperglycaemia. Excessive gluconeogenesis in the liver contributes to hyperglycaemia ${ }^{(16)}$, and chronically elevated blood glucose has a significant impact on the liver itself. Diabetic patients have been found to have excessive glycogen deposition in the liver ${ }^{(17)}$ and excessive build-up of fat in the liver as steatosis ${ }^{(18)}$. More recently, Almon et al. ${ }^{(19)}$ compared gene expression patterns in the liver of Wistar and GK rats and found that 311 genes are differentially expressed in the liver of the two strains. A functional analysis of these genes indicated that disruption of lipid metabolism in the liver is a major consequence of chronic hyperglycaemia in the GK strain and that chronic inflammation contributes significantly to the development of diabetes in GK rats. In the present study, the effects of treatment with mitochondrial nutrients on mitochondrial function, oxidative damage and apoptosis in the liver of GK rats are presented, and compared with those of pioglitazone treatment.

\section{Materials and methods}

\section{Animals and treatments}

Male diabetic GK rats, 4 weeks old, together with age-matched male non-diabetic Wistar rats were purchased from SLAC Laboratory Animal Company Limited (Shanghai, China). All animals were housed at $23 \pm 2^{\circ} \mathrm{C}$ under $12 \mathrm{~h}$ light $-12 \mathrm{~h}$ dark cycles, and allowed access to food and water ad libitum. National Institutes of Health ${ }^{(20)}$ principles of laboratory animal care were strictly followed, and all experimental procedures were approved by the Institutional Animal Use and Care of the Institute for Nutritional Science, Shanghai Institutes of Biological Sciences.

Rats were divided into four groups (each group, $n$ 10): (1) untreated Wistar and (2) GK control groups, (3) a pioglitazone group that received pioglitazone $(20 \mathrm{mg} / \mathrm{kg}$ per d) by oral administration, (4) a nutrient group that received a combination of $R$ - $\alpha$-LA $\quad(50 \mathrm{mg} / \mathrm{kg}$ per $\mathrm{d})$, acetyl-L-carnitine $(100 \mathrm{mg} / \mathrm{kg}$ per $\mathrm{d})$, biotin $(0 \cdot 1 \mathrm{mg} / \mathrm{kg}$ per $\mathrm{d})$ and nicotinamide $(15 \mathrm{mg} / \mathrm{kg}$ per $\mathrm{d})$ by oral administration. The control groups received the same oral administration volume of PBS alone. The treatments started at 6 weeks of age, and continued for 3 months. At approximately 18 weeks of age, animals were anaesthetised with an intraperitoneal injection of sodium pentobarbital $(60 \mathrm{mg} / \mathrm{kg})$ and killed in order to obtain the liver.

\section{Isolation of liver mitochondria and liver tissue protein}

Liver mitochondria were isolated as described previously ${ }^{(21)}$ with a slight modification ${ }^{(22,23)}$. Mitochondrial protein concentration was determined using a Pierce $\mathrm{BCA}^{\mathrm{TM}}$ Protein Assay kit (product no. 23225; Thermo Scientific, Rockford, IL, USA), with bovine serum albumin as a standard. Mitochondria were stored at $-80^{\circ} \mathrm{C}$ until enzyme analysis.

For tissue protein collection, approximately $100 \mathrm{mg}$ of liver samples were homogenised in ice-cold solubilisation buffer containing $65 \mathrm{~mm}$-Tris ( $\mathrm{pH} 7 \cdot 4$ ), $150 \mathrm{~mm}-\mathrm{NaCl}, 5 \mathrm{~mm}$-EDTA, $1 \%$ Nonidet P-40, 0.5\% sodium deoxycholate, $0.1 \%$ SDS, $10 \%$ glycerol, aprotinin $(1 \mu \mathrm{g} / \mathrm{ml})$, leupeptin $(1 \mu \mathrm{g} / \mathrm{ml}), 10 \mathrm{~mm}$ sodium fluoride, $1 \mathrm{~mm}-\mathrm{Na}_{3} \mathrm{VO}_{4}$ and $1 \mathrm{~mm}$-phenylmethylsulfonylfluoride. Protein concentrations were determined as described earlier.

\section{Assays of complexes I, II, III, IV and V activities in mitochondria}

The activities of the mitochondrial complexes NADHcoenzyme quinone (CoQ) oxidoreductase (complex I), succinate-CoQ oxidoreductase (complex II), CoQ-cytochrome $c$ reductase (complex III), cytochrome $c$ oxidase (complex IV) and ATP synthase (complex V) were measured spectrometrically as described previously ${ }^{(24-26)}$. 
Assays for activities of total antioxidant capacity, superoxide dismutase, glutathione S-transferase, $N A D(P) H$ :quinone oxidoreductase 1 and reduced glutathione in mitochondria

Total antioxidant capacity was assayed with a commercially available assay kit (Jiancheng Biochemical, Inc., Nanjing, China). The principle of the test is to measure the change in colour upon conversion of $\mathrm{Fe}^{3+}$ to $\mathrm{Fe}^{2+}$ by the reducing components in the samples. Optical density was measured at $520 \mathrm{~nm}$ with a microplate reader. Total superoxide dismutase activity was assayed using a commercially available SOD Detection Kit (Jiancheng Biochemical, Inc.). The assay is based on the superoxide generated by the xanthine/xanthine oxidase system. Optical density at $550 \mathrm{~nm}$ was measured with a microplate reader. Glutathione $S$-transferase activity was measured with $2 \mu \mathrm{g}$ protein in a final $200 \mu \mathrm{l}$ volume of $1 \mathrm{~mm}$-reduced glutathione (GSH), $1 \mathrm{~mm}$-chloro-2,4-dinitrobenzene, and bovine serum albumin $(3 \mathrm{mg} / \mathrm{ml})$ in $10 \mathrm{~mm}$-sodium phosphate buffer. The mixture was scanned at $340 \mathrm{~nm}$ for $5 \mathrm{~min}$ at $25^{\circ} \mathrm{C}$ as described previously ${ }^{(27)}$.

$\mathrm{NAD}(\mathrm{P}) \mathrm{H}$ :quinone oxidoreductase 1 activity was measured with $10 \mu \mathrm{g}$ protein in a final $200 \mu \mathrm{l}$ volume of $50 \mathrm{~mm}$-Tris$\mathrm{HCl}$ (pH 7.4), $80 \mu \mathrm{m}$-dichlorophenolindophenol (DCPIP), bovine serum albumin $(1 \mathrm{mg} / \mathrm{ml}), 0.005 \%$ Tween-20 (v/v), and 0 or $10 \mu \mathrm{m}$-dicoumarol; the reaction was initiated with $180 \mu \mathrm{m}-\mathrm{NADPH}$. The reduction of DCPIP was scanned at $600 \mathrm{~nm}$ at $25^{\circ} \mathrm{C}$ with a microplate reader. $\mathrm{NAD}(\mathrm{P}) \mathrm{H}$ :quinone oxidoreductase 1 activity was considered to be the dicoumarol-inhibitable part of the DCPIP reduction.

GSH content was determined in the liver with a commercially available GSH Detection Kit (Jiancheng Biochemical, Inc.) that utilises a thiol-specific reagent, dithionitrobenzoic acid. The adduct was measured spectrophotometrically at $412 \mathrm{~nm}$

\section{Western blot of glyceraldehyde 3-phosphate} dehydrogenase in mitochondria

Liver mitochondrial protein samples $(10 \mu \mathrm{g})$ were subjected to $10 \%$ SDS-PAGE; proteins were then transferred to nitrocellulose membranes and blocked with 5\% non-fat milk/TBST (Tris-buffered saline Tween 20) for $1 \mathrm{~h}$ at room temperature. Membranes were incubated with primary monoclonal antibodies directed against glyceraldehyde 3-phosphate dehydrogenase (GAPDH, 1:1000) overnight at $4^{\circ} \mathrm{C}$. Western blots were developed using enhanced chemiluminescence (Roche, Mannheim, Germany) and quantified by scanning densitometry. As mitochondrial protein loading controls, polyacrylamide resolving gels $(10 \%, \mathrm{w} / \mathrm{v})$ loaded with the same samples were stained with Coomassie Brilliant Blue R250.

\section{Detection of mitochondrial protein carbonyls}

Protein carbonyls in liver mitochondria were assayed with the Oxyblot protein oxidation detection kit (Chemicon, Temecula, CA, USA) ${ }^{(24,28)}$.

\section{Western blot analysis of p53 and p21}

Liver tissue proteins were subjected to 10\% SDS-PAGE and detected with primary antibodies against p53 (1:1000, Mouse Ab no. 12506; Santa Cruz Biotechnology, Santa Cruz, CA, USA), p21 (1:1000, Mouse Ab no. 2946; Cell Signaling Technology, Danvers, MA, USA) or $\alpha$-tubulin (1:5000). Western blots were developed using enhanced chemiluminescence (Roche) and quantified by scanning densitometry (Bio-Rad Laboratories, Hercules, CA, USA) after incubation with horseradish peroxidase-conjugated secondary antibody.

\section{Determination of NEFA contents of Goto-Kakizaki liver tissue}

NEFA contents of supernatants were estimated by a commercially available NEFA kit (Jiancheng Biochemical, Inc.). The test is based on the reaction of NEFA with $\mathrm{Cu}^{2+}$ to form Cu salts, which are detected photometrically at $440 \mathrm{~nm}$.

\section{RNA isolation and RT-PCR for PPAR $\alpha$ and carnitine palmitoyltransferase I}

Total RNA was isolated using the single-step TRI-reagent (TRIzol), and $1 \mu \mathrm{g}$ of isolated RNA was then reversetranscribed into complementary DNA. Primer sequences used for quantification of mRNA by real-time quantitative PCR for $\operatorname{PPAR} \alpha$, carnitine palmitoyltransferase I (CPT-1), and $18 \mathrm{~S}$ ribosomal RNA and mRNA have been described previously ${ }^{(29)}$.

\section{Statistical analysis}

Data are presented as means with their standard errors. Statistical significance was calculated with SPSS 10.0 software (SPSS Inc., Chicago, IL, USA) using one-way ANOVA. $P$ values $<0.05$ were considered significant.

\section{Results}

\section{Activities of liver mitochondrial complexes}

As shown in Fig. 1, all five complexes composing the respiratory chain were assayed photometrically. Compared with Wistar controls, activities of complexes I, III and IV distinctly decreased in the liver mitochondria of GK rats $(P<0 \cdot 01)$. Conversely, the activities of complexes II and $\mathrm{V}$ distinctly increased in GK rats. The nutrient treatment significantly prevented the decreases in complexes I, III and IV $(P<0.05)$; in contrast, treatment with pioglitazone produced no significant improvement. As for complexes II and V, nutrient treatment showed no significant changes compared with GK untreated rats, while pioglitazone treatment significantly increased the activity of complex V.

\section{Liver mitochondrial protein carbonyl levels}

As shown in Fig. 2, protein carbonyl levels were higher in the liver mitochondria of GK rats compared with those of control rats $(P<0 \cdot 01)$. Nutrient treatment significantly diminished 


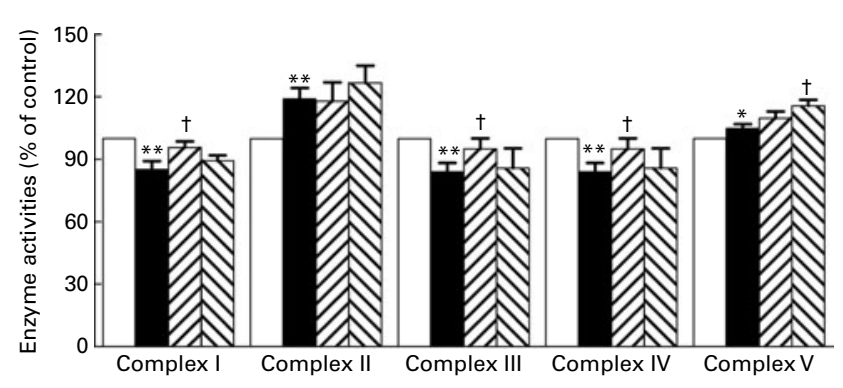

Fig. 1. Liver mitochondrial activities of complexes I-V. The complex activities were determined as described in Materials and methods. Results are expressed as fold change of control. Values are means, with their standard errors represented by vertical bars $(n 10)$. Mean value was significantly different from that of the Wistar group: ${ }^{*} P<0.05$, ${ }^{* *} P<0.01$. † Mean value was significantly different from that of the untreated Goto-Kakizaki (GK) group $(P<0.05)$. $\square$, Wistar; $\mathbf{\square}, \mathrm{GK} ; \square$, GK with nutrients; $\mathbb{}$, GK with pioglitazone.

the carbonyls in liver mitochondria compared with GK rats $(P<0.05)$, while in the pioglitazone group, there was only a tendency for carbonyl levels to be lowered in liver mitochondria.

\section{Total antioxidant capacity, reduced g/utathione contents} and activities of total superoxide dismutase, glutathione S-transferase and $N A D(P) H$ :quinone oxidoreductase 1

The total antioxidant capacity of the GK rat liver was distinctly reduced (Fig. 3(a)), and treatment of nutrients significantly elevated it; however, the pioglitazone group did not show a significant increase. In contrast, the GSH level in the GK rat liver unexpectedly increased, and treatment with nutrients and pioglitazone tended to normalise it (Fig. 3(a)). Compared with the Wistar control mitochondria, activities of total superoxide dismutase, glutathione $S$-transferase and $\mathrm{NAD}(\mathrm{P}) \mathrm{H}$ : quinone oxidoreductase 1 were significantly reduced in GK rats $(P<0.05)$, and the nutrient-treated group demonstrated significant changes as shown in Fig. 3(b).
Protein expression of glyceraldehyde 3-phosphate dehydrogenase in liver mitochondria

GAPDH protein expression was significantly increased in the liver mitochondria of untreated GK rats compared with the expression in Wistar rat mitochondria $(P<0.05)$; the nutrient and pioglitazone groups demonstrated significant normalisation of protein expression compared with the GK group $(P<0 \cdot 05)$, as shown in Fig. 4.

Protein expression of the apoptosis-related factors, p53 and p21 in liver tissue

p53 protein expression was significantly increased in the liver tissue of GK rats (Fig. 5) compared with that in Wistar rats, and the increase in p53 was accompanied by an increased expression of $\mathrm{p} 21$ protein. Nutrient treatment tended to inhibit the increase in $\mathrm{p} 53$ and $\mathrm{p} 21$, while treatment with pioglitazone did not produce a significant difference.

\section{NEFA content in the liver}

As shown in Fig. 6, NEFA content was higher in the liver of diabetic GK rats than that in Wistar rats; treatment with nutrients significantly decreased the NEFA level $(P<0 \cdot 05)$.

\section{PPAR $\alpha$ and CPT-1 gene expression changes in liver tissue}

Levels of PPAR $\alpha$ mRNA were lower in GK rats, although the difference was not statistically significant. Administration of nutrients to GK rats significantly increased the expression of PPAR $\alpha$ levels (Fig. 7). In contrast, levels of CPT-1 mRNA were significantly lower in GK rats than in Wistar rats $(P<0.05)$. Administration of either nutrients or pioglitazone to GK rats significantly increased the levels of CPT- 1 in the GK rat liver (Fig. 7).

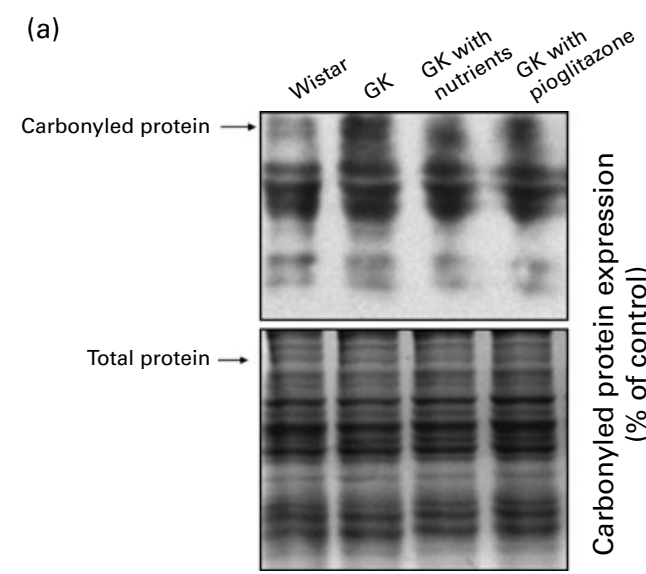

Fig. 2. Carbonylated protein levels in liver mitochondria. Liver protein carbonyls were detected by Western blotting using the Oxyblot protein oxidation detection kit (Chemicon, Temecula, CA, USA). The second set of polyacrylamide resolving gels $(12 \%$, w/v) loaded with the same quantity of samples was electrophoresed and stained with Coomassie Brilliant Blue R250 as a loading control. (a) Western blotting images of carbonylated protein; (b) quantitative results of carbonylated protein. Quantitative values were computed as the ratio of band densities of the respective protein to total balanced protein. Results are expressed as fold change of control. Values are means, with their standard errors represented by vertical bars $(n 10) .{ }^{* *}$ Mean value was significantly different from that of the Wistar group $(P<0.01)$. † Mean value was significantly different from that of the untreated Goto-Kakizaki (GK) group $(P<0.05)$. $\square$, Wistar; $\mathbf{\square}, \mathrm{GK} ; \mathbb{Z}, \mathrm{GK}$ with nutrients; $\mathbb{\nabla}, \mathrm{GK}$ with pioglitazone. 

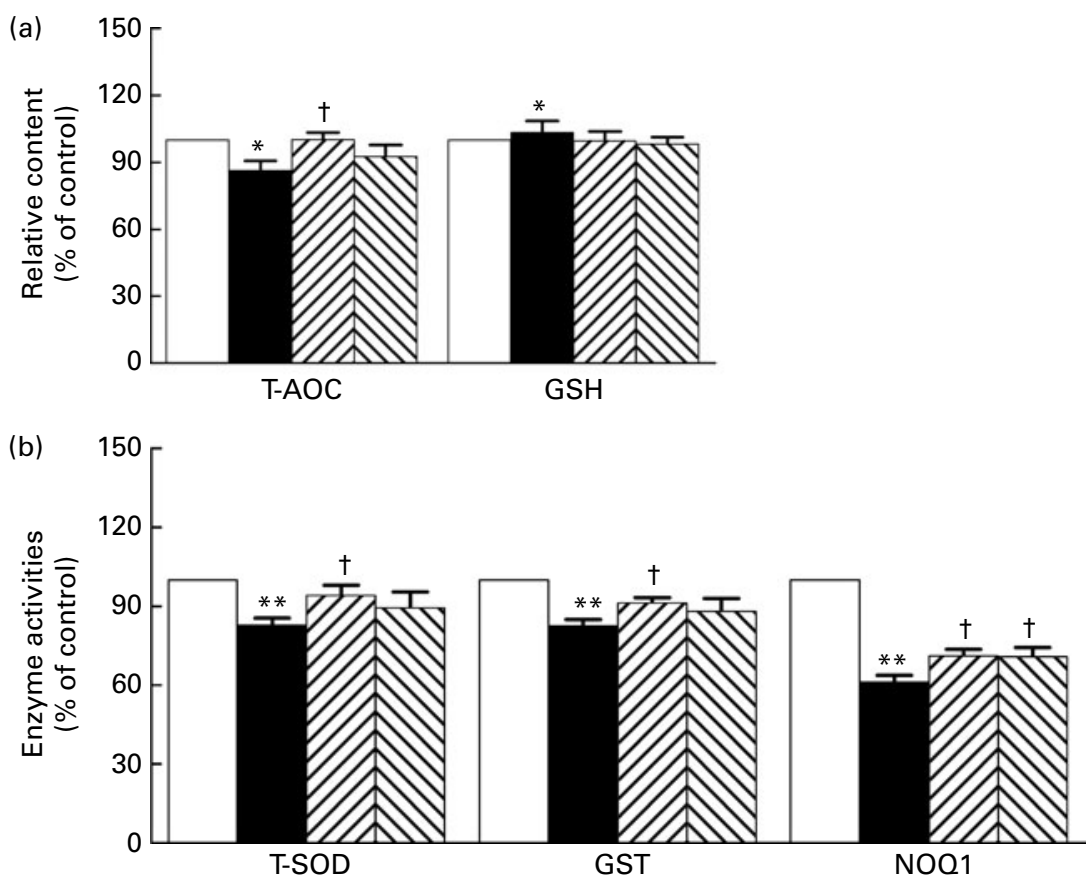

Fig. 3. Liver mitochondrial total antioxidant capacity (T-AOC), contents of reduced glutathione (GSH) and activities of total superoxide dismutase (T-SOD), glutathione S-transferase (GST) and NAD(P)H:quinone oxidoreductase 1 (NQO1). (a) T-AOC and GSH in mitochondria ( $\square$, Wistar; $\mathbf{m}$, Goto-Kakizaki (GK); $\square$, GK with

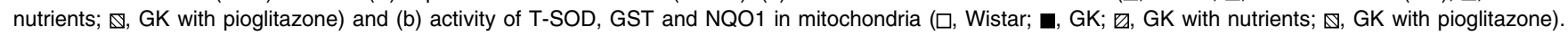
Results are expressed as fold change of control. Values are means, with their standard errors represented by vertical bars $(n 10)$. Mean value was significantly different from that of the Wistar group: ${ }^{\star} P<0.05,{ }^{\star \star} P<0.01$. † Mean value was significantly different from that of the untreated GK group $(P<0.05)$.

\section{Discussion}

As with $\beta$-cells, the liver is highly exposed to the possibility of glucose injury (glucose toxicity). Emerging evidence supports the hypothesis that insulin responsiveness of the liver in type 2 diabetes is caused by mitochondrial dysfunction ${ }^{(5)}$. It has been shown that liver mitochondrial function appears to be more efficient and that the liver has higher levels of antioxidants such as vitamin E, GSH and CoQ in 6-month-old GK rats than in non-diabetic control rats ${ }^{(30-33)}$. It is difficult to understand why GK rat liver mitochondria function at high efficiency while operating under the stressful conditions of diabetic hyperglycaemia. One possible explanation for over-reactive mitochondria and high antioxidant response is a diabetes-induced metabolic maladaptation ${ }^{(33)}$.

In the present study, liver mitochondrial function is significantly altered in GK rats, as indicated by the decreases in complex I, III and IV activities and increases in complex II and $\mathrm{V}$ activities. Treatment with the combination of mitochondrial nutrients, more significantly than with pioglitazone, normalised the activity of liver mitochondrial complexes in GK rats towards that in non-diabetic Wistar rats, suggesting that the mitochondrion is a possible target of these nutrients. That complexes I, III and IV all respond similarly is consistent with other characteristics they have in common: the activities of all three are inhibited by $\mathrm{NO}^{(34)}$, critical subunits of all three are encoded by mitochondrial genes (in contrast to complex II) and all three act to pump protons out of the mitochondrial matrix (in contrast to complex V). The increases in complexes II and $\mathrm{V}$ are consistent with a previous report by Ferreira et $a l .{ }^{(31)}$ but the changes in complexes I and IV are different. Ferreira et al. ${ }^{(31)}$ showed that complex I and complexes II and III did not change while complex IV increased. The lack of change of complexes II and III in Ferreira et al.'s work may be due to an increase in complex II combined with a decrease in complex III, but the reasons for the differences in complexes I and IV between the present study and Ferreira et al.'s are unknown. The only difference between the two studies is that in the present study, nutrients were administered by oral administration, whereas Ferreira et al. used animals without any treatment. Whether stress induced by oral administration caused a decrease in the activity of complexes I and IV warrants further study.

GSH, CoQ and $\alpha$-tocopherol are important endogenous antioxidants, and are generally decreased by oxidative insult. GK rat liver mitochondria have increases in mitochondrial $\mathrm{CoQ}^{(33)}$, vitamin $\mathrm{E}$ ( $\alpha$-tocopherol) contents and reduced: oxidised glutathione ratios $^{(30)}$, possibly in response to increased oxidative stress. In agreement with these results, liver GSH levels in GK rats were significantly increased, compared with those in Wistar rats. Treatments with nutrients or pioglitazone reversed the GSH increase by a similar amount, suggesting that they may play a role in relieving hyperglycaemia-induced oxidative stress in the liver. However, it should be pointed out that the lack of the measurement of oxidised glutathione is a limitation in the present study.

GAPDH expression was prominently higher in GK rats, compared with that in Wistar rats (Fig. 4). GAPDH was first characterised as a glycolytic dehydrogenase, but it has been shown since to exert several functions as diverse as apoptosis 


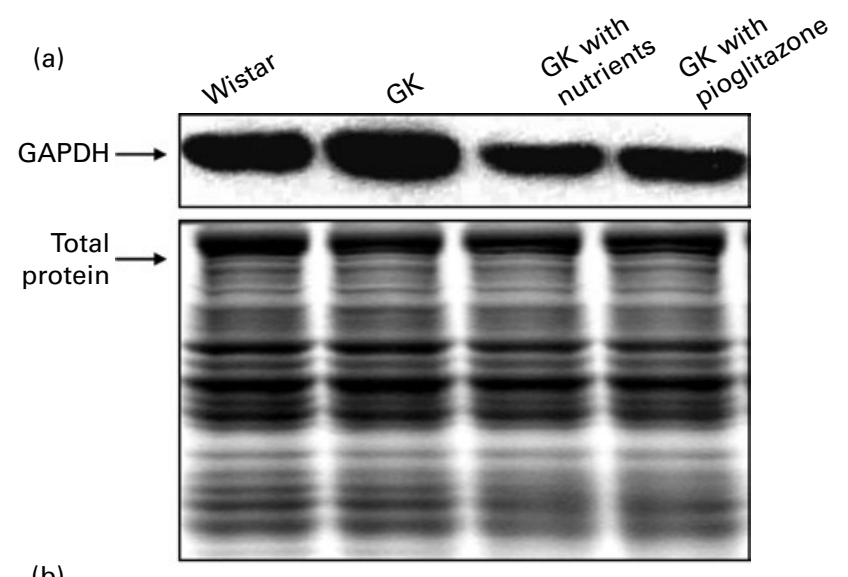

(b)

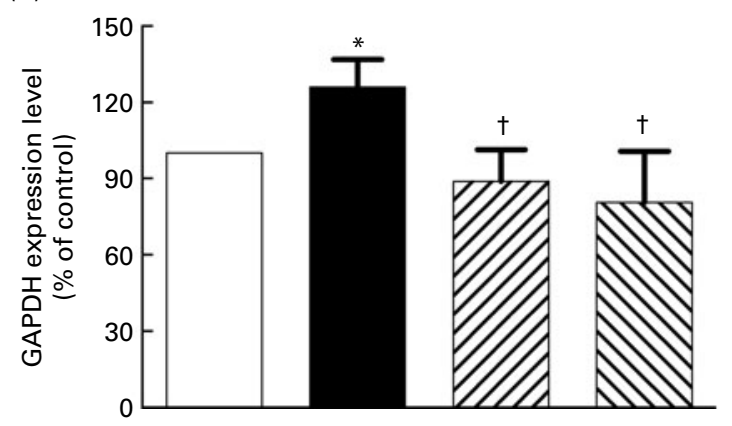

Fig. 4. Protein expression of glyceraldehyde 3-phosphate dehydrogenase (GAPDH) in liver mitochondria: (a) Western blotting images of GAPDH protein and (b) quantitative results for GAPDH. Quantitative values were computed as the ratios of the density of the targeted protein to total balanced protein. Results are expressed as fold change of control. Values are means, with their standard errors represented by vertical bars $(n 10) .{ }^{*}$ Mean value was significantly different from that of the Wistar group $(P<0.05)$. $†$ Mean value was significantly different from that of the untreated Goto-Kakizaki (GK) group

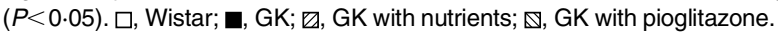

induction, transfer RNA export, DNA repair or as a receptorassociated kinase ${ }^{(35)}$. It is a pleiotropic enzyme that is overexpressed during apoptosis and in several human chronic pathologies. This protein accumulates in mitochondria during apoptosis, and regulates pro-apoptotic mitochondrial membrane permeabilisation, a decisive event in the intrinsic pathway of apoptosis. This latter functionality provided motivation to investigate protein expression of the apoptosisregulating factors $\mathrm{p} 53$ and $\mathrm{p} 21$. Consistent with the elevation of GAPDH and mitochondrial dysfunction, levels of both p53 and p21 proteins were elevated - the first report of increased expression of these factors in the GK rat liver. Treatment with either the nutrient combination or pioglitazone significantly decreased GAPDH expression (Fig. 4). These treatments also produced a non-significant decrease in p53 and p21 (Fig. 5), consonant with the statistically significant reduction in these two proteins seen in GK rat thymocytes reported earlier ${ }^{(15)}$.

As evidence indicating a possible increase in oxidative stress and consequent cell death/tissue damage in GK rat mitochondria, amounts of oxidised proteins were clearly elevated in GK rats, and total antioxidant capacity, total superoxide dismutase, glutathione $S$-transferase and $\mathrm{NAD}(\mathrm{P}) \mathrm{H}$ :quinone oxidoreductase 1 activities were substantially decreased. These results suggest that hyperglycaemia-induced metabolic maladaptation will consequently cause oxidative damage in GK rats. Treatment of GK rats with mitochondrial nutrients efficiently prevented oxidative damage in liver. These results are in line with the improvement of impaired glucose tolerance and hyperinsulinaemia ${ }^{(14)}$, and closely parallel similar ameliorative effects of these nutrients on the same oxidative parameters in GK rat thymocytes ${ }^{(15)}$.

NEFA content in diabetic GK rat plasma is substantially elevated $^{(36)}$, although GK rats demonstrate neither severe dyslipidaemia nor fatty liver. In the present study, NEFA content in the liver tissue of GK animals increased significantly compared with that of Wistar animals, and nutrient treatment significantly ameliorated this increase (Fig. 6). This pattern parallels previously reported results in plasma ${ }^{(14)}$. Nutrient treatment also affected the expression of hepatic genes related to lipid oxidation pathways that are altered in GK animals. Expression of PPAR $\alpha$ and CPT-1 gene expression decreased in the GK rat liver. PPAR $\alpha$ and CPT-1 regulate the mitochondrial and peroxisomal fatty acid $\beta$-oxidation systems as well as the microsomal $\omega$-oxidation system. Defects in these factors have been reported to lead to defects in the utilisation of hepatic fat as an energy source and consequently to hypoketonaemia that has a prominent impact on the pathogenesis of fatty liver disease ${ }^{(37,38)}$. In the GK model, NEFA accumulation was suppressed by mitochondrial nutrient treatment along with significant up-regulation of PPAR $\alpha$ and CPT-1 mRNA
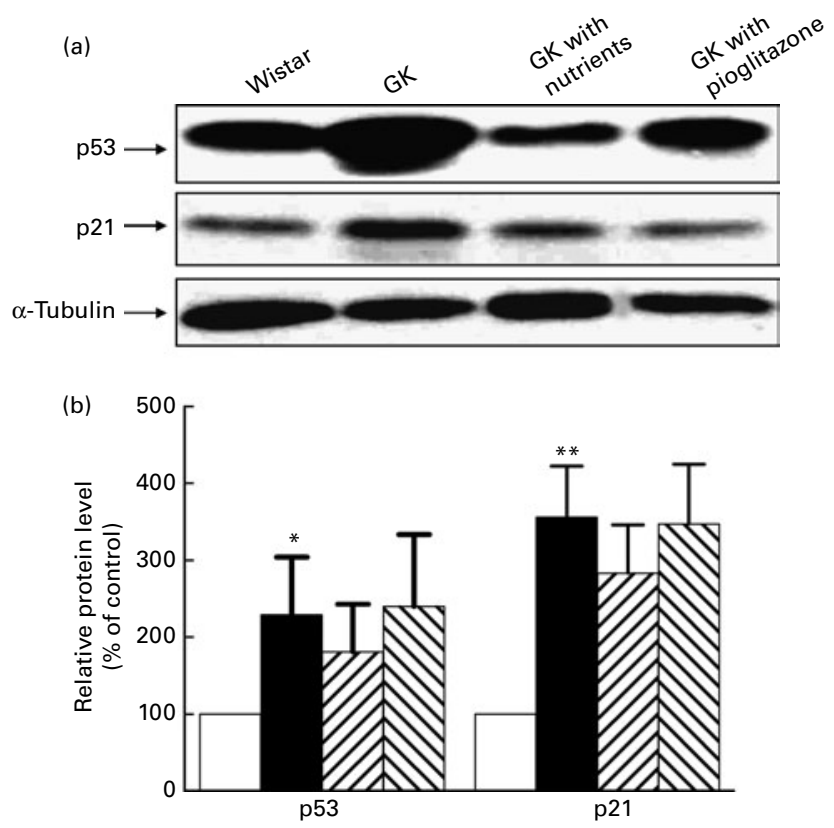

Fig. 5. Apoptosis-related factors p53 and p21 in liver tissue: (a) Western blotting images of p21, p53 and $\alpha$-tubulin, respectively and (b) quantitative results of $p 53$ and $\mathrm{p} 21$. Quantitative values were computed as the ratios of the density of the targeted protein to $\alpha$-tubulin. Results are expressed as fold change of control. Values are means, with their standard errors represented by vertical bars $(n 10)$. Mean value was significantly different from that of the Wistar group: * $P<0.05,{ }^{\star *} P<0.01$. $\square$, Wistar; $\mathbf{\square}, \mathrm{GK}$; $\square$, GK with nutrients; $\mathbb{\nabla}$, GK with pioglitazone. 


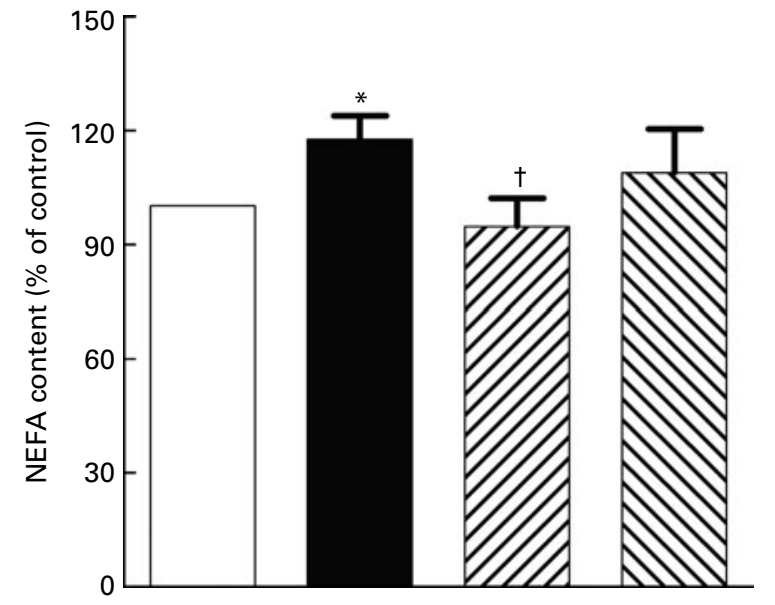

Fig. 6. NEFA content in liver tissue. For tissue protein collection, approximately $50 \mathrm{mg}$ of liver samples were homogenised in ice-cold buffer as described in Materials and methods. After centrifugation, the supernatant was used for NEFA detection. Results are expressed as percentage of control. Values are means, with their standard errors represented by vertical bars. * Mean value was significantly different from that of the Wistar group $(P<0.05)$. † Mean value was significantly different from that of the untreated Goto-Kakizaki (GK) group $(P<0.05)$. $\square$, Wistar; $\square$, GK; $\square$, GK with nutrients;

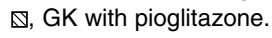

transcription, indicating that mitochondrial nutrients decreased NEFA contents by up-regulating lipid oxidation pathways. In GK rat muscle, comparable decreases in PPAR $\alpha$ and CPT-1 gene expression levels that were restored to wild-type levels by nutrient treatment suggest that similar metabolic alterations may occur in the latter tissue as well ${ }^{(14)}$

In summary, GK rat livers differ from Wistar controls by displaying abnormal changes in the activities of mitochondrial complexes, an increase in oxidative stress, a decrease in antioxidant defences and increases in NEFA levels and apoptosis. Furthermore, a 3-month treatment with a combination of four nutrients ameliorated these abnormalities to the same degree

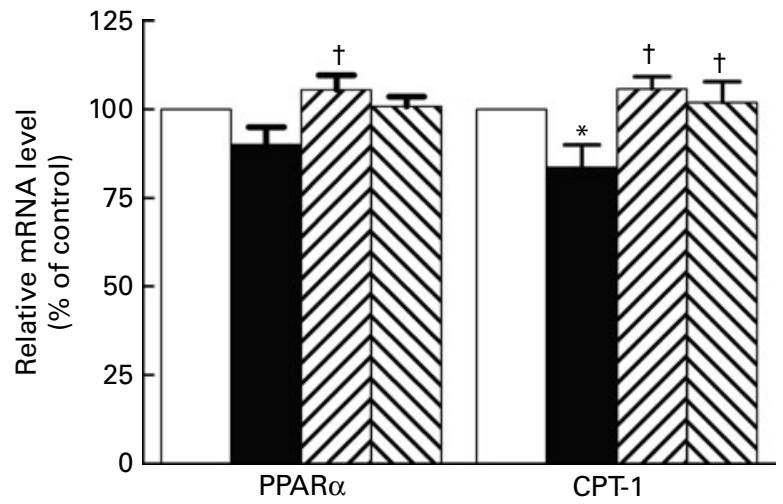

Fig. 7. Gene expression of PPAR $\alpha$ and CPT-1, two genes involved in fatty acid oxidation in liver tissue. PCR fluorescence products were quantified using SYBR Green. The cycle number at which the various transcripts were detectable was compared with that of $18 \mathrm{~S}$ ribosomal RNA as an internal control. Results are expressed as percentage of control. Values are means, with their standard errors represented by vertical bars. * Mean value was significantly different from that of the Wistar group $(P<0 \cdot 05)$. $†$ Mean value was significantly different from that of the untreated Goto-Kakizaki (GK) group $(P<0.05)$. $\square$, Wistar; $\mathbf{\square}$, GK; $\square$, GK with nutrients; $\mathbb{}$, GK with pioglitazone. as did treatment with the antidiabetic drug pioglitazone. These results suggest that dietary supplementation with these nutrients may be an effective strategy to improve liver dysfunction in GK diabetic rats by improving mitochondrial function, inhibiting oxidative damage and reducing apoptosis.

\section{Acknowledgements}

The authors thank Xu Jia, Lu Zhu, Cheng Luo and Xuesen Li for technical assistance. The present study was supported by Pujiang Talent Award (05PG14104), the Open Research Fund Program of Key Laboratory of Marine Drugs Ministry of Education, Ocean University of China, a grant from the Shanghai Science and Technology Committee (074319105), Shanghai, China, the National Natural Science Foundation of China Grant (30871002), a UC Davis Center for Human and Nutrition Pilot Award (CHNR08-318) and the financial support of 985 and 211 projects of Xi'an Jiaotong University and the National Natural Science Foundation of China (grant no. 31070740). J. H., W. S., X. S. and J. L. conceived and designed the experiments; J. H., W. S., L. S. and J. L. performed the experiments; J. H., W. S. and J. L. analysed the data; J. H., E. S. and J. L. wrote the manuscript. A patent (application no. 200710046615.8) has been filed on the combination of nutrients mentioned in the study. The authors declare that no competing interests exist.

\section{References}

1. Biessels GJ, Kappelle AC, Bravenboer B, et al. (1994) Cerebral function in diabetes mellitus. Diabetologia 37 , 643-650.

2. McCarty MF (1997) Exploiting complementary therapeutic strategies for the treatment of type II diabetes and prevention of its complications. Med Hypotheses 49, 143-152.

3. Zimmet PZ (1995) The pathogenesis and prevention of diabetes in adults. Genes, autoimmunity, and demography. Diabetes Care 18, 1050-1064.

4. Ames BN, Shigenaga MK \& Hagen TM (1995) Mitochondrial decay in aging. Biochim Biophys Acta 1271, 165-170.

5. Lowell BB \& Shulman GI (2005) Mitochondrial dysfunction and type 2 diabetes. Science 307, 384-387.

6. Cai X, Jia H, Liu Z, et al. (2008) Polyhydroxylated fullerene derivative $\mathrm{C}(60)(\mathrm{OH})(24)$ prevents mitochondrial dysfunction and oxidative damage in an $\mathrm{MPP}(+)$-induced cellular model of Parkinson's disease. J Neurosci Res 86, 3622-3634.

7. Liu J, Shen W, Zhao B, et al. (2009) Targeting mitochondrial biogenesis for preventing and treating insulin resistance in diabetes and obesity: hope from natural mitochondrial nutrients. Adv Drug Deliv Rev 61, 1343-1352.

8. Fuchs J, Packer L \& Zimmer G (1997) Lipoic Acid in Health and Disease. New York: Marcel Dekker, Inc.

9. Liu J (2008) The effects and mechanisms of mitochondrial nutrient alpha-lipoic acid on improving age-associated mitochondrial and cognitive dysfunction: an overview. Neurochem Res 33, 194-203.

10. Janssen U, Vassiliadou A, Riley SG, et al. (2004) The quest for a model of type II diabetes with nephropathy: the Goto Kakizaki rat. J Nephrol 17, 769-773.

11. Portha B, Lacraz G, Kergoat M, et al. (2009) The GK rat betacell: a prototype for the diseased human beta-cell in type 2 diabetes? Mol Cell Endocrinol 297, 73-85. 
12. Palmeira CM, Ferreira FM, Santos DL, et al. (1999) Higher efficiency of the liver phosphorylative system in diabetic Goto-Kakizaki (GK) rats. FEBS Lett 458, 103-106.

13. Duhault J \& Koenig-Berard E (1997) Diabetes mellitus and its animal models. Therapie 52, 375-384.

14. Shen W, Hao J, Tian C, et al. (2008) A combination of nutriments improves mitochondrial biogenesis and function in skeletal muscle of type 2 diabetic Goto-Kakizaki rats. PLoS One 3, e2328.

15. Hao J, Shen W, Tian C, et al. (2009) Mitochondrial nutrients improve immune dysfunction in the type 2 diabetic Goto-Kakizaki rats. J Cell Mol Med 13, 701-711.

16. Gastaldelli A, Baldi S, Pettiti M, et al. (2000) Influence of obesity and type 2 diabetes on gluconeogenesis and glucose output in humans: a quantitative study. Diabetes 49 , 1367-1373.

17. Clore JN, Post EP, Bailey DJ, et al. (1992) Evidence for increased liver glycogen in patients with noninsulindependent diabetes mellitus after a 3-day fast. J Clin Endocrinol Metab 74, 660-666.

18. Lonardo A, Lombardini S, Ricchi M, et al. (2005) Review Article: hepatic steatosis and insulin resistance. Aliment Pharmacol Ther 22, Suppl. 2, 64-70.

19. Almon RR, DuBois DC, Lai W, et al. (2009) Gene expression analysis of hepatic roles in cause and development of diabetes in Goto-Kakizaki rats. J Endocrinol 200, 331-346.

20. NIH (1985) Principles of laboratory animal care. NIH publication no. 85-23. http://grants1.nih.gov/grants/olaw/ references/phspol.htm

21. Krahenbuhl S, Chang M, Brass EP, et al. (1991) Decreased activities of ubiquinol:ferricytochrome $c$ oxidoreductase (complex III) and ferrocytochrome c:oxygen oxidoreductase (complex IV) in liver mitochondria from rats with hydroxycobalamin[c-lactam]-induced methylmalonic aciduria. $J$ Biol Chem 266, 20998-21003.

22. Long J, Wang X, Gao H, et al. (2007) D-Galactose toxicity in mice is associated with mitochondrial dysfunction: protecting effects of mitochondrial nutrient $R$-alpha-lipoic acid. Biogerontology 8, 373-381.

23. Long J, Gao H, Sun L, et al. (2009) Grape extract protects mitochondria from oxidative damage and improves locomotor dysfunction and extends lifespan in a Drosophila Parkinson's disease model. Rejuvenation Res 12, 321-331.

24. Sun L, Luo C, Long J, et al. (2006) Acrolein is a mitochondrial toxin: effects on respiratory function and enzyme activities in isolated rat liver mitochondria. Mitochondrion 6, 136-142.

25. Li X, Liu Z, Luo C, et al. (2008) Lipoamide protects retinal pigment epithelial cells from oxidative stress and mitochondrial dysfunction. Free Radic Biol Med 44, 1465-1474.
26. Jia L, Liu Z, Sun L, et al. (2007) Acrolein, a toxicant in cigarette smoke, causes oxidative damage and mitochondrial dysfunction in RPE cells: protection by $(R)$-alpha-lipoic acid. Invest Ophthalmol Vis Sci 48, 339-348.

27. Pabst MJ, Habig WH \& Jakoby WB (1974) Glutathione $S$-transferase A. A novel kinetic mechanism in which the major reaction pathway depends on substrate concentration. J Biol Chem 249, 7140-7147.

28. Levine RL, Garland D, Oliver CN, et al. (1990) Determination of carbonyl content in oxidatively modified proteins. Methods Enzymol 186, 464-478.

29. Shen W, Liu K, Tian C, et al. (2008) R-alpha-lipoic acid and acetyl-L-carnitine complementarily promote mitochondrial biogenesis in murine 3T3-L1 adipocytes. Diabetologia 51, 165-174.

30. Ferreira FM, Palmeira CM, Matos MJ, et al. (1999) Decreased susceptibility to lipid peroxidation of Goto-Kakizaki rats: relationship to mitochondrial antioxidant capacity. Life Sci 65, 1013-1025.

31. Ferreira FM, Palmeira CM, Seica R, et al. (2003) Diabetes and mitochondrial bioenergetics: alterations with age. J Biochem Mol Toxicol 17, 214-222.

32. Ferreira FM, Palmeira CM, Seica R, et al. (1999) Alterations of liver mitochondrial bioenergetics in diabetic Goto-Kakizaki rats. Metabolism 48, 1115-1119.

33. Ferreira FM, Seica R, Oliveira PJ, et al. (2003) Diabetes induces metabolic adaptations in rat liver mitochondria: role of coenzyme Q and cardiolipin contents. Biochim Biophys Acta 1639, 113-120.

34. Carreras MC, Franco MC, Peralta JG, et al. (2004) Nitric oxide, complex I, and the modulation of mitochondrial reactive species in biology and disease. Mol Aspects Med 25, 125-139.

35. Tarze A, Deniaud A, Le Bras M, et al. (2007) GAPDH, a novel regulator of the pro-apoptotic mitochondrial membrane permeabilization. Oncogene 26, 2606-2620.

36. Bogacka I, Xie H, Bray GA, et al. (2005) Pioglitazone induces mitochondrial biogenesis in human subcutaneous adipose tissue in vivo. Diabetes 54, 1392-1399.

37. Reddy JK \& Rao MS (2006) Lipid metabolism and liver inflammation. II. Fatty liver disease and fatty acid oxidation. Am J Physiol Gastrointest Liver Physiol 290, G852-G858.

38. Hashimoto T, Cook WS, Qi C, et al. (2000) Defect in peroxisome proliferator-activated receptor alpha-inducible fatty acid oxidation determines the severity of hepatic steatosis in response to fasting. J Biol Chem 275, 28918-28928. 